\title{
Folate receptor-targeted fluorescent paramagnetic bimodal liposomes for tumor imaging
}

This article was published in the following Dove Press journal:

International Journal of Nanomedicine

19 October 2011

Number of times this article has been viewed

\author{
Nan Ding ${ }^{1,2}$ \\ Yao Lu' \\ Robert J Lee ${ }^{3}$ \\ Chang Yang' \\ Lei Huang' \\ Jian Liu' \\ Guangya Xiang ${ }^{1,4}$
}

'School of Pharmacy, Tongji Medical College, Huazhong University of Science and Technology, Wuhan, People's Republic of China;

${ }^{2}$ Department of Pharmacy, Tongji Hospital, Tongji Medical College, Huazhong University of Science and Technology, Wuhan, People's Republic of China; ${ }^{3}$ Division of Pharmaceutics, College of Pharmacy, The Ohio State University, Columbus, Ohio, USA; ${ }^{4}$ Hubei Key Laboratory of Natural Medicinal Chemistry and Resource Evaluation, Tongji Medical College, Huazhong University of Science and Technology, Wuhan, People's Republic of China

The first three authors contributed equally to this work.

Correspondence: Guangya Xiang School of Pharmacy, Tongji Medical College, Huazhong University of Science and Technology, Hangkong

Road 13, Wuhan, 430030,

People's Republic of China

Tel +862783692793

Fax +862783692793

Email gyxiang1968@hotmail.com
Rationale and objective: Receptor-targeted delivery of imaging and therapeutic agents can lead to enhanced efficacy for both. Multimodality imaging offers unique advantages over traditional single modality imaging. Tumor marker folate receptor (FR)-targeted fluorescent paramagnetic bimodal liposomes were synthesized to co-deliver paramagnetic and fluorescence agents for magnetic resonance (MR) and optical bimodal imaging contrast enhancement.

Materials and methods: Fluorescent and paramagnetic bimodal liposomes were synthesized with a mean diameter of $136 \mathrm{~nm}$ and a low polydispersity index. The liposomes incorporated folate-PEG ${ }_{3350}$-CHEMS for FR targeting, Gd(III)[N,N-Bis-stearylamidomethyl$\mathrm{N}$ '-amidomethyl]diethylenetriamine tetraacetic acid (Gd-DTPA-BSA) for MR contrast, and calcein for fluorescence. To determine the specificity and efficiency of delivery, the liposomes were evaluated in FR-positive KB and HeLa cells and FR-negative A549 cells, which were analyzed by fluorescence microscopy, magnetic resonance imaging (MRI), and flow cytometry (FCM).

Results: FR-specific and efficient cellular uptake of the FR-targeted bimodal liposomes was confirmed by fluorescence microscopy and by FCM. The mean fluorescence intensity (MFI) of $\mathrm{KB}$ cells treated with FR-targeted liposomes was $45 \times$ that of cells treated with nontargeted liposomes, and $18 \times$ that of cells treated with FR-targeted liposomes and excess folic acid (FA). The MFI of HeLa cells treated with targeted liposomes was $33 \times$ that of nontargeted liposomes, and was $16 \times$ that of the mixture of targeted liposomes and free FA. In contrast, the MFI of A549 cells treated with FR-targeted liposomes was nearly the same as those treated with nontargeted liposomes. The $\mathrm{T}_{1}$-weighted MR images of HeLa and KB cells incubated with FR-targeted liposomes had much higher signal intensity than those treated with nontargeted liposomes or free Gd-DTPA. Furthermore, the FR-targeting effect could be blocked by excess free FA.

Conclusion: FR-targeted fluorescent paramagnetic bimodal liposomes provided a novel platform for bimodal tumor imaging and theranostic delivery.

Keywords: folate receptor, tumor targeting, MRI, fluorescence, bimodal liposomes

\section{Introduction}

Noninvasive diagnostic imaging techniques such as magnetic resonance imaging (MRI) and optical tomography are becoming increasingly important in cancer diagnosis and therapy. Imaging contrast agents can facilitate tumor detection at tissue, cellular and molecular levels. ${ }^{1}$ MRI enables the visualization of the three-dimensional structure of tissues. It has a high degree of spatial resolution but suffers from a relatively low sensitivity. Contrast agents can offer useful signal enhancement to MRI. ${ }^{2}$ Gadoliniumdiethylenetriamine pentaacetic acid/dimeglumine (Gd-DTPA/dimeglumine) 
(Magnevist $^{\circledR}$, Bayer HealthCare, Leverkusen, Germany) is the first US Food and Drug Authority-approved clinical contrast agent. It is a low molecular weight compound without tissue specificity that is cleared rapidly from the circulation. Several contrast agents have been developed to prolong the circulation time and improve the tissue specificity of the magnetic resonance (MR) contrast agent. ${ }^{3-6}$ For example, Gd(III) [N,N-Bis-stearylamidomethyl-N'-amidomethyl] diethylenetriamine tetraacetic acid (Gd-DTPA-BSA), an amphipathic Gd complex, has been synthesized for incorporation into liposomes. ${ }^{7}$ The resulting paramagnetic liposomes effectively enhanced $\mathrm{T}_{1}$-imaging. Optical imaging is another useful technology platform. Fluorescent probes can be used as optical contrast agents. However, optical imaging is hampered by the very limited depth of light penetration. ${ }^{8}$ Combining MRI and fluorescence imaging would enable visualization of tumors by MRI and analysis of histological samples by fluorescence imaging. ${ }^{9,10}$ In addition, Kircher et al reported the delineation of tumors both by preoperative MRI and by intraoperative optical imaging. ${ }^{11}$

Lipid-based nanoparticles, such as liposomes, micelles, and microemulsions, have been evaluated as carriers of MRI contrast and fluorescent agents for noninvasive imaging. ${ }^{9,12-20}$ There are several recent reviews on lipid-based paramagnetic nanoparticles ${ }^{21,22}$ and on paramagnetic and fluorescent nanoparticles..$^{3,10,23,24}$ Kostarelos and Miller described a modular design for targeted paramagnetic liposomes carrying nucleic acids, termed Gd-ABCD. ${ }^{25}$ These are Gd liposomes, in which " $\mathrm{A}$ " is condensed nucleic acids. " $\mathrm{B}$ " is a lipid bilayer, " $\mathrm{C}$ " is the PEG polymer, and " $\mathrm{D}$ " is a suitable ligand. ${ }^{25}$

Folate receptor (FR) is a well-known tumor marker. It has limited expression in normal tissues, and is greatly overexpressed in a variety of carcinomas. ${ }^{26}$ Folate conjugates of imaging agents have high binding affinity for cellsurface FRs, which allows for selective targeting of tumor cells. ${ }^{27}$ Kamaly et al showed that FR-targeted fluorescent paramagnetic bimodal liposomes have increased accumulation in a FR + murine tumor model. ${ }^{13}$

In this study, the authors report the synthesis and evaluation of FR-targeting bimodal liposomes for MRI and fluorescent imaging based on the previously reported Gd-ABCD design. ${ }^{25}$ Gd-DTPA-BSA was incorporated into the bilayer of the liposomes as a paramagnetic agent, whereas calcein, a fluorescent dye, was encapsulated in the aqueous core of liposomes. Folate-polyethylene glycol $_{3350^{-}}$ cholesteryl hemisuccinate (folate- $\mathrm{PEG}_{3350}-\mathrm{CHEMS}^{28}$ was used as a ligand for FR targeting. FR-mediated tumor cell targeting by these bimodal liposomes was demonstrated both by fluorescence imaging and MRI.

\section{Materials and methods Materials}

Hydrogenated soy phosphatidylcholine (HSPC) and monomethoxy polyethylene glycol $_{2000}$-distearoylphosphatidylethanolamine $\left(\mathrm{mPEG}_{2000}\right.$-DSPE) were purchased from Avanti Polar Lipids Inc (Alabaster, AL). Cholesterol and octadecylamine were obtained from J\&K Chemical Ltd (Beijing, China). Sepharose CL-4B chromatography media was purchased from Sigma Aldrich (St Louis, MO). Folic acid was purchased from Huixing Biological and Chemical Reagents Co, Ltd (Shanghai, China). Gadolinium oxide was purchased from Sinopharm Chemical Reagent Co, Ltd (Shanghai, China). Diethylenetriaminepentaacetic acid (DTPA) was purchased from Tianjin Yuanhang Chemical Reagents Co, Ltd (Tianjin, China). Calcein was purchased from Aladdin Reagent Co (Shanghai, China). Folate-PEG ${ }_{3350}{ }^{-}$ CHEM was synthesized in the authors' laboratory, as described previously. ${ }^{28}$ All reagents were of analytical grade and were used without any further purification.

L-glutamine, trypsin $(1: 250)$, and EDTA-Na ${ }_{2}$ were purchased from Amresco (Solon, OH). Penicillin-streptomycin (100,000 units/mL) was supplied by Hyclone (Logan, UT). Fetal bovine serum (FBS) was a product of Hangzhou Sijiqing Biological Engineering Materials Co, Ltd (Hangzhou, China). Roswell Park Memorial Institute medium (RPMI)-1640 10× medium without folic acid was purchased from Sigma Aldrich.

The human nasopharyngeal epidermal carcinoma KB cell line, which has been identified as being derived from the human cervical cancer HeLa cell line, was obtained from the Cell Bank at the Chinese Academy of Science (Shanghai, China). The human cervical carcinoma HeLa cell line was provided by the Tongji Hospital, Tongji Medical College, Huazhong University of Science and Technology, Hubei, China. A549, a human lung carcinoma cell line, was provided by the Union Hospital, Tongji Medical College.

\section{Synthesis of Gd-DTPA-BSA}

[N,N-Bis-stearylamidomethyl-N'-amidomethyl]diethylenetriamine tetraacetic acid (DTPA-BSA) was prepared using the method outlined by Kamaly et al. ${ }^{14}$ Briefly, a solution of octadecylamine dissolved in chloroform was added to a solution of DTPA bis-anhydride previously dissolved in dimethylformamide. The reaction was stirred at $40^{\circ} \mathrm{C}$ 
for 2 hours. The white precipitate formed was collected by filtration and dried under vacuum overnight. The white solid was stirred in water to dissolve any excess DTPA and stirred in boiling chloroform to dissolve any excess DTPA bis-anhydride and octadecylamine. $\mathrm{Gd}^{3+}$ was then added to synthesize Gd-DTPA-BSA according to the procedure of Kabalka et al. ${ }^{7}$ The concentrations of Gd were determined by inductively coupled plasma atomic emission spectroscopy (ICP-AES) (OPTIMA 4300DV, Perkin Elmer Ltd, Waltham, MA). The phase-transition temperature of Gd-DTPA-BSA was determined by differential scanning calorimetry on a Diamond DSC (Perkin Elmer Ltd, Waltham, MA).

\section{Bimodal liposome preparation}

The FR-targeted liposomes were prepared by hydration of a thin lipid film composed of $\mathrm{HSPC} / \mathrm{mPEG}_{2000}$-DSPE/ cholesterol/Gd-DTPA-BSA/ folate-PEG ${ }_{3350}$-CHEMS at 30/40/25/4/1 (mole/mole). Nontargeted liposomes composed of $\mathrm{HSPC} / \mathrm{mPEG}_{2000}$-DSPE/Cholesterol/Gd-DTPA-BSA $30 / 40 / 25 / 5$ (mole/mole) were synthesized by the same method. Briefly, the lipids $(0.05 \mathrm{mmol}$ total $)$ were dissolved in chloroform/methanol (1/1), followed by evaporation to dryness to form a lipid film. The lipid film was subsequently hydrated in $2 \mathrm{~mL} 2.5 \mathrm{mM}$ calcein solution for 40 minutes at $60^{\circ} \mathrm{C}$ and then sonicated for 10 minutes. The resulting lipid dispersion was extruded sequentially five times through polycarbonate membrane filters with a pore diameter of $200 \mathrm{~nm}$ and $100 \mathrm{~nm}$ (Lipex Extruder, Northern Lipids Inc, Vancouver, Canada). Liposomes and free calcein were separated by size exclusion chromatography on a Sepharose CL-4B gel (Sigma Aldrich).

The size and zeta potential of the liposomes were determined by dynamic light scattering on a Zetasizer (nanoseries) Nano-ZS 90 (Malvern Instruments Ltd, Malvern, Worcestershire, UK). Fluorescence measurements were carried out on a fluorescence spectrophotometer (LS55 fluorescence spectrophotometer, Perkin Elmer Ltd). The incorporation of Gd in the liposome preparations was confirmed by ICP-AES. For MR imaging, nontargeted liposomes (L-Gd/calcein), FR-targeted liposomes (F-L-Gd/calcein), and Gd-DTPA were prepared at three different concentrations. The MR images of liposome suspensions were collected, along with the MR image of water. The $\mathrm{T}_{1}$-weighted image was acquired by $3.0 \mathrm{~T}$ MRI on a Signa HDxt Hdi 3.0T MRI (General Electric, Fairfield, CT).

\section{In vitro targeting experiments}

\section{Fluorescence imaging of cells}

$\mathrm{KB}$ cells were plated in a six-well plate at $37^{\circ} \mathrm{C}$ under $5 \%$ $\mathrm{CO}_{2}$ in folate-free RPMI 1640 medium containing $10 \%$ fetal bovine serum. The cells were grown until $80 \%$ confluence was reached. The medium was then removed and replaced with fresh medium (without serum). F-L-Gd/calcein, L-Gd/ calcein, and F-L-Gd/calcein plus $1 \mathrm{mM}$ folic acid (FA) were added. FA was added to FR-targeted liposomes prior to use in cellular treatment. All liposomes contained calcein at a concentration of $15.2 \mu \mathrm{M}$. One mM FA was used as an FR blocking agent. After incubation at $37^{\circ} \mathrm{C}$ for 1 hour, the cells were examined on an Olympus IX71 fluorescence microscope (Perkin Elmer Ltd).

\section{Quantitative analysis of cell uptake}

$\mathrm{KB}, \mathrm{HeLa}$, and A549 cells were seeded into six-well plates and separately incubated with F-L-Gd/calcein, L-Gd/calcein, and F-L-Gd/calcein plus $1 \mathrm{mM}$ FA. The final concentration of calcein in the incubation media was $12 \mu \mathrm{M}$. After incubation at $37^{\circ} \mathrm{C}$ for 1 hour, the cells were washed $3 \times$ with cold phosphate-buffered saline (PBS) and treated with trypsin. The fluorescence intensity of cells was analyzed by flow cytometry (FCM) on a BD-LSR cytometer (BD Biosciences, Franklin Lakes, NJ). Each study was repeated 3-4 times.

\section{MR imaging of cells}

$\mathrm{HeLa}$ and KB cells were grown in folate-free RPMI 1640 medium containing 10\% fetal bovine serum. The cells were grown to $80 \%$ confluence and were then suspended by treatment with trypsin. Aliquots of the cells were transferred to Eppendorf tubes and were incubated with fresh media containing FR-targeted or nontargeted liposomes. The final concentration of Gd in the incubation media was $18.5 \mathrm{mg} / \mathrm{L}$. After incubation at $37^{\circ} \mathrm{C}$ for 1 hour, cells were washed $3 \times$ with cold PBS. The tubes were imaged on a 3.0T MRI with a standard spin-echo sequence (time of repetition $=20,40,100$, $200 \mathrm{~ms}$; time of echo $=9.0 \mathrm{~ms} / \mathrm{Fr}$; number of excitation: 2.0 ).

\section{Results \\ Synthesis of Gd-DTPA-BSA}

The identity of Gd-DTPA-BSA product was confirmed by its melting point and IR spectrum (data not shown). Based on ICPAES determination, the complex contained $13.3 \% \mathrm{Gd}$. The phase-transition temperature of Gd-DTPA-BSA was found to be $65.32^{\circ} \mathrm{C}$. 


\section{Characterization of the bimodal liposomes}

\section{Size and zeta potential of the liposomes}

Liposomes had a mean diameter of $\sim 136 \mathrm{~nm}$ with a low polydispersity index. The preparations were stable for at least 3 months at $4^{\circ} \mathrm{C}$ (Figure 1A). The zeta potential of liposomes was $\sim-13.6 \mathrm{mV}$ (Figure 1B).

\section{The incorporation of calcein and Gd}

The maximum fluorescence emission wavelength of calcein did not change when being encapsulated in liposomes (Ex-max was at $491 \mathrm{~nm}$, Em-max was at $511 \mathrm{~nm}$ ). The encapsulation efficiency for calcein was low, ranging from $1.2 \%$ to $4.7 \%$, as determined by UV absorption at $491 \mathrm{~nm}$. Meanwhile, the encapsulation efficiency for Gd was substantially higher, ranging from $20 \%$ to $27 \%$, as determined by ICP-AES.

\section{MR imaging of liposomes}

Compared with water, the Gd liposomes and Gd-DTPA all showed brighter MR signals in $\mathrm{T}_{1}$-weighted images (Figure 2). Therefore, the paramagnetic liposomes were demonstrated to be MR detectable. The intensity of the signal was concentration dependent. A decrease in the concentration of the Gd liposomes was associated with a reduction in signal intensity in the MR image (Figure 2). The MR images of Gd-DTPA were slightly brighter than those of liposomes. This might be due to easier proton exchange between the bulk water and the Gd-DTPA.

\section{In vitro targeting experiments Fluorescence imaging of cells}

Uptake of liposomes by FR positive KB cells was analyzed by fluorescence microscopy. Green fluorescence was associated with liposomal calcein. As shown in Figure 3, F-L-Gd/calcein (Figure 3B and Figure 3B') was taken up by the KB cells more efficiently than nontargeted liposomes (Figure 3A and Figure 3A'), and the uptake could be blocked by $1 \mathrm{mM}$ free FA (Figure $3 \mathrm{C}$ and Figure $3 \mathrm{C}^{\prime}$ ). Additionally, FR-targeted liposomes were primarily located on the surface of cell membrane. This phenomenon was consistent with previous observations, ${ }^{29,30}$ which showed extensive surface binding of FR-targeted liposomes to the FR on the cell surface.

\section{Quantitative analysis of cell uptake}

Targeted and nontargeted liposomes were incubated with FR positive tumor cells (KB and HeLa) and FR negative tumor cells (A549). Free FA, used as a blocking agent, was added to FR-targeted liposomes prior to use in cellular treatment. The FR specificity of cellular uptake of targeted and nontargeted liposomes was quantitatively analyzed by FCM. The experiments were repeated at least three times. (Table 1, Figure 4). The MFI of KB cells treated with F-L-Gd/calcein was 45 times higher than that of cells treated with nontargeted L-Gd/calcein, and 18 times higher than that of cells treated with the F-L-Gd/ calcein plus $1 \mathrm{mM}$ free FA. The MFI of HeLa cells treated with F-L-Gd/calcein was 33 times higher than that of cells treated with nontargeted liposomes L-Gd/calcein, and 16 times higher than that of cells treated with the F-L-Gd/calcein plus $1 \mathrm{mM}$ free FA. In contrast, the MFI of A549 cells treated with F-L-Gd/calcein was nearly identical with that of cells treated with L-Gd/calcein, or F-L-Gd/calcein plus free FA. In summary, the FR-targeted liposomes F-L-Gd/ calcein showed delivery specificity in FR positive cells but not in FR negative cells, and the FR targeting effect could be blocked by $1 \mathrm{mM}$ free FA.

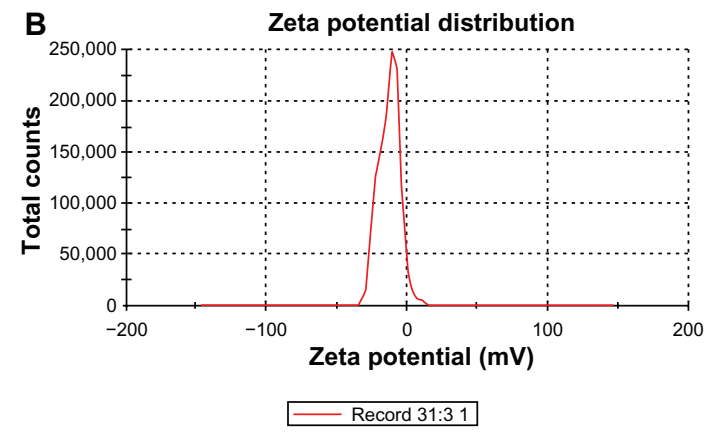

Figure I Size (A) and zeta potential (B) distribution of the FR-targeted liposomes (F-L-Gd/calcein). The mean diameter of the liposomes was I36 nm (A), and the zeta potential was about $-13.6 \mathrm{mV}(\mathbf{B})$.

Abbreviation: $F R$, folate receptor. 


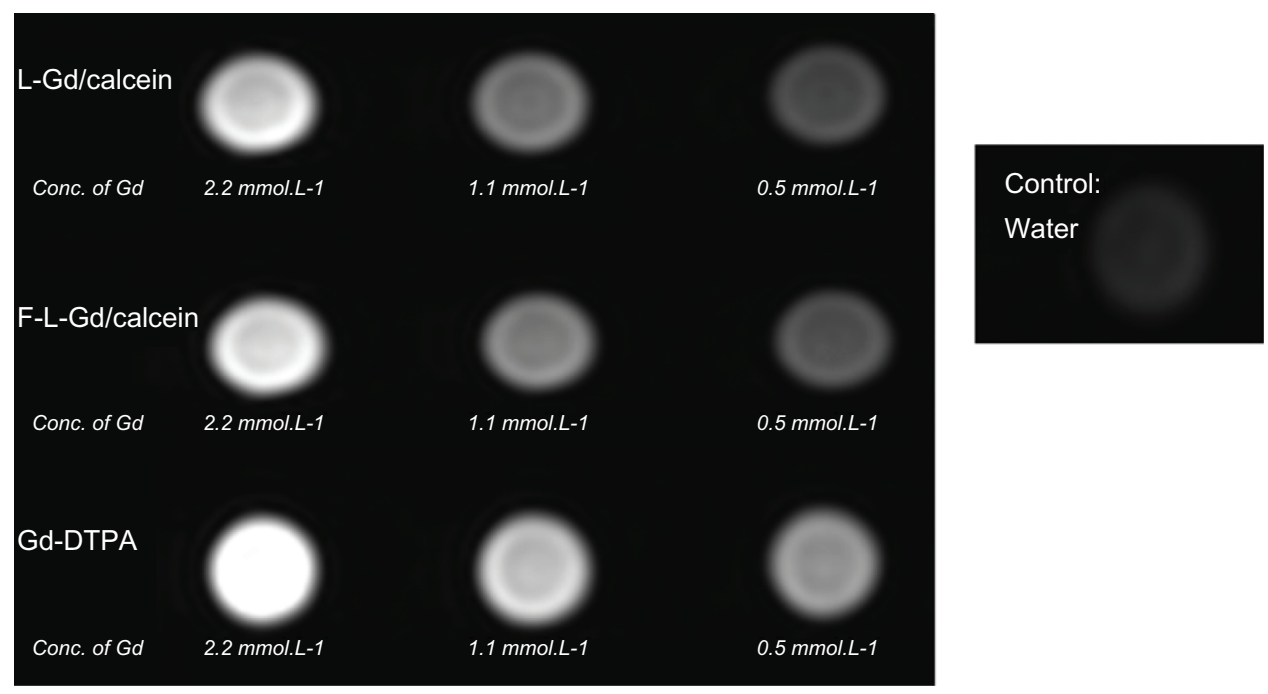

Figure $2 \mathrm{~T}_{1}$-weighted images of Gd-liposomes and Gd-DTPA.

Abbreviations: DTPA, diethylenetriamine pentaacetic acid; Conc., concentration.

\section{MRI of cells}

For MRI, HeLa cells were incubated with (1) blank liposome, (2) L-Gd/calcein, (3) F-L-Gd/calcein, (4) F-L-Gd/ calcein +1 mM FA, or (5) Gd-DTPA for 1 hour. From $\mathrm{T}_{1}$-weighted images, the tube containing HeLa cells incubated with F-L-Gd/calcein (Figure 5C) had a much higher signal intensity than that of the tubes of cells treated with blank liposomes or nontargeted liposomes (Figure 5A and 5B). This was probably due to FR-mediated uptake by the FR-positive HeLa cells. The MR signal was diminished when the cells were incubated with F-L-Gd/calcein + 1 mM FA. This showed the blocking of FR-dependent uptake by free FA (Figure 5D).
The MR image of HeLa cells incubated with F-L-Gd/calcein was also brighter than that of HeLa cells incubated with GdDTPA (Figure 5E). This may be due to the fact that Gd-DTPA was unable to enter cells by diffusion due to its hydrophilicity and was therefore removed during washing of the cells. Meanwhile, the liposomes were retained on the cell surface via FR binding and therefore enhanced the $\mathrm{T}_{1}$ signal.

KB cells were also incubated with (1) L-Gd/calcein, (2) F-L-Gd/calcein, or (3) F-L-Gd/calcein + 1 mM FA for 1 hour at $37^{\circ} \mathrm{C}$ followed by washing with PBS. MRI showed that cells incubated with F-L-Gd/calcein had the highest signal intensity (Figure 6B), and that this could be blocked
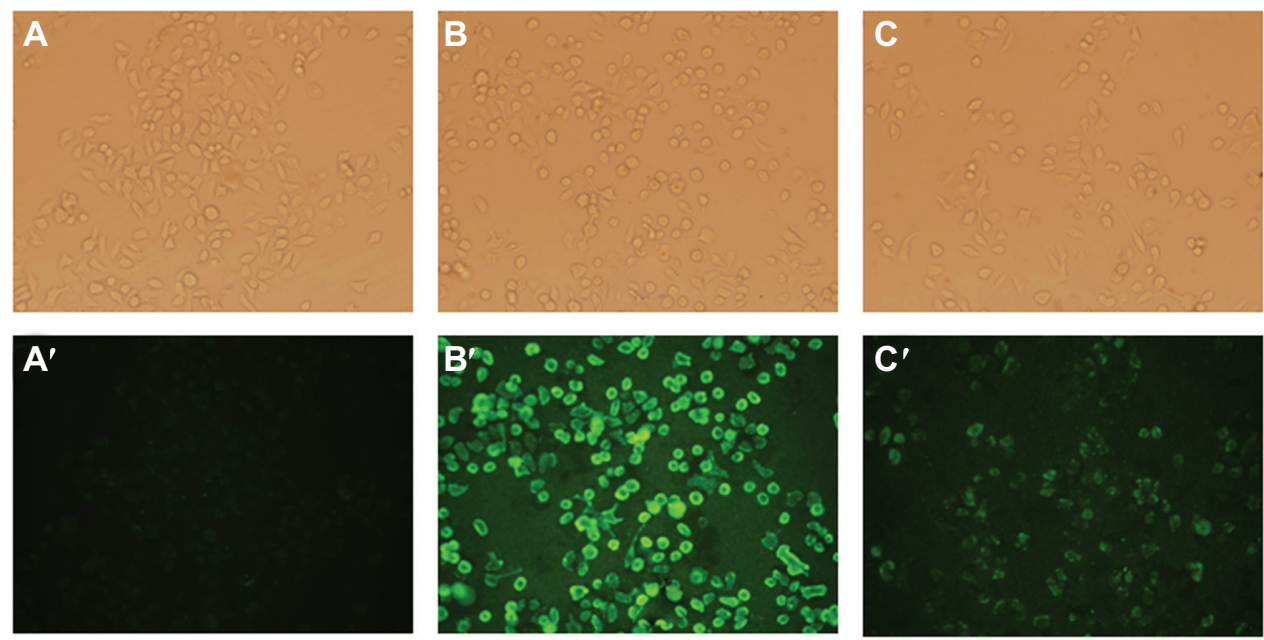

Figure 3 Uptake of liposomal calcein by KB cells. The cells were incubated at $37^{\circ} \mathrm{C}$ for I hour with L-Gd/calcein $\left(\mathbf{A}, \mathbf{A}^{\prime}\right)$, F-L-Gd/calcein $\left(\mathbf{B}, \mathbf{B}^{\prime}\right)$, or F-L-Gd/calcein + I mM FA (C, $\left.\mathbf{C}^{\prime}\right)$. Top panels show cells visualized in the phase-contrast mode (A, B, C); bottom panels show the same fields in the fluorescence mode ( $\mathbf{A}^{\prime}$, $\left.\mathbf{B}^{\prime}, \mathbf{C}^{\prime}\right)$ Abbreviation: FA, folic acid. 
Table I Uptake of liposomal calcein by KB, HeLa, and A549 cells determined by flow cytometry

\begin{tabular}{lccc}
\hline & L-Gd/calcein & F-L-Gd/calcein & F-L-Gd/calcein + FA \\
\hline KB & $491 \pm 236$ & $22,334 \pm 8451$ & $1269 \pm 395$ \\
HeLa & $96 \pm 20$ & $3181 \pm 400$ & $203 \pm 120$ \\
A549 & $110 \pm 22$ & $138 \pm 82$ & $151 \pm 104$ \\
\hline
\end{tabular}

Abbreviation: FA, folic acid.

by $1 \mathrm{mM}$ free FA (Figure 6C). These data further confirmed FR-dependent cellular uptake of F-L-Gd/calcein in another FR-positive cell line.

\section{Discussion}

Liposomes are long-circulating vehicles that are capable of encapsulation of water-soluble agents and incorporation of amphiphilic and hydrophobic agents in the lipid bilayer. Two types of liposomal MRI contrast agents have been described. ${ }^{22,23}$ The first is based on liposomes entrapping paramagnetic agents, typically Gd-DTPA, in the aqueous lumen. ${ }^{31,32} \mathrm{~A}$ drawback of these liposomes is that encapsulation reduces the relaxivity of the paramagnetic agent, because of the limited access to exchange with bulk water by the contrast agents. A second type of liposomal contrast agents are based on liposomes carrying amphiphilic paramagnetic molecules in the lipid bilayer, which makes them an integral part of the liposomal surface, therefore providing greater access to proton exchange with water compared with the liposomes of the first type. Lipid chain length and degree of saturation affect the particle relaxivity of the second type of liposomes. ${ }^{33}$

In this study, a combination of an amphiphilic GdDTPA-BSA and a fluorescent dye, calcein, was used to prepare fluorescent paramagnetic bimodal liposomes. Gd-DTPA-BSA has been used in previous studies. ${ }^{9,19}$ The

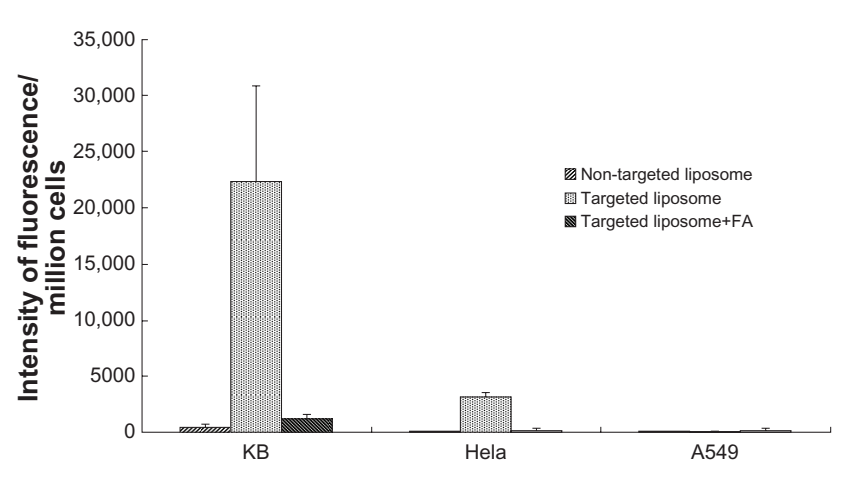

Figure 4 Comparison of the uptake of liposomes between FR-positive (KB, HeLa) and FR-negative (A549) cell lines.

Abbreviations: FR, folate receptor; FA, folic acid.

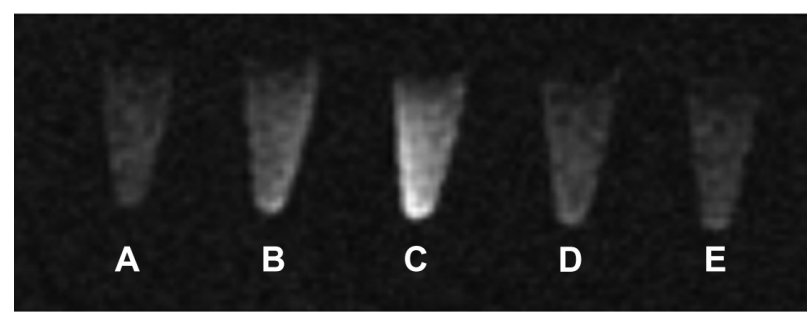

Figure $\mathbf{5} \mathrm{T}_{1}$-weighted image of HeLa cells after incubated with contrast agents (coronal scan). (A) L. (B) L-Gd/calcein. (C) F-L-Gd/calcein. (D) F-L-Gd/calcein + I mM FA. (E) Gd-DTPA.

Abbreviations: FA, folic acid; DTPA, diethylenetriamine pentaacetic acid.

amount of Gd-DTPA-BSA used in this study, which was 25 mole $\%$ of total lipids $(50 \mu \mathrm{mol})$, was consistent with previous reports. ${ }^{9}$ Calcein is a hydrophilic fluorescent marker that has been used to investigate membrane permeability. ${ }^{9}$ Calcein can be replaced by quantum dots, other fluorescent probes, or a hydrophilic therapeutic drug in further work. Effective FR targeting was achieved by incorporation of folate- $\mathrm{PEG}_{3350}-\mathrm{CHEM}$ at $1 \mathrm{~mole} \%$ of the total lipids.

The small size, the slightly negative zeta potential of the liposomes (Figure 1), along with surface PEGylation are expected to produce a relatively long circulation time in vivo. ${ }^{21,34}$ The liposomes were shown to be paramagnetic (Figure 2). When the concentration of the contrast agents decreased, the contrast of MR images was also decreased. The $T_{1}$ image intensity of Gd-liposomes and Gd-DTPA were much higher than that of water. In addition, the $\mathrm{T}_{1}$ images of Gd-DTPA were slightly brighter than those of Gd-liposomes at the same Gd concentration, presumably due to greater access to proton exchange with environmental water.

The uptake of F-L-Gd/calcein by KB and HeLa cells was FR specific, which was also confirmed qualitatively by fluorescence microscopy (Figure 3) and MRI (Figure 5, Figure 6), and then quantitatively by FCM (Table 1, Figure 4). FR-targeted liposomes were primarily located on the surface
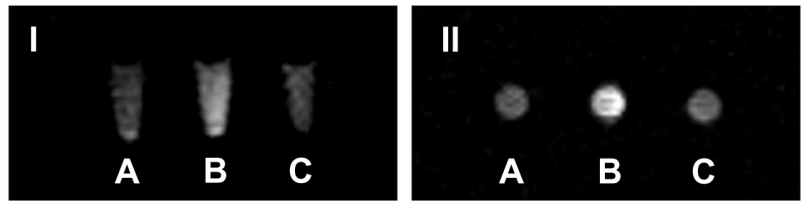

Figure $6 T_{1}$-weighted image of $K B$ cells after incubated with contrast agents (I, coronal scan; II, axial scan) (A) L-Gd/calcein. (B) F-L-Gd/calcein. (C) F-L-Gd/ calcein + I mM FA.

Abbreviation: FA, folic acid. 
of the cell membrane (Figure 3), due to binding to FR. Uptake of the liposomes was greater in KB cells than in HeLa cells. This can be explained by the higher FR expression in the former. ${ }^{35}$

\section{Conclusion}

FR-targeted fluorescent paramagnetic bimodal liposomes were synthesized. Both MRI and fluorescence imaging showed FR-specific uptake of these liposomes in FR-positive but not FR-negative cells. Further work will determine the plasma clearance kinetics and the biodistribution of the liposomes in vivo. In addition, the authors will explore loading the liposomes with therapeutic agents to enable image-guided therapy.

\section{Acknowledgments}

This work was supported by National Natural Science Foundation of China grant (No. 81072596), Program for New Century Excellent Talents in University (No. NCET-080226), and the Fundamental Research Funds for the Central Universities (HUST: M2009044).

\section{Disclosure}

The authors report no conflicts of interest in this work.

\section{References}

1. Sumer B, Gao J. Theranostic nanomedicine for cancer. Nanomedicine (Lond). 2008;3(2):137-140.

2. Frias JC, Ma Y, Williams KJ, Fayad ZA, Fisher EA. Properties of a versatile nanoparticle platform contrast agent to image and characterize atherosclerotic plaques by magnetic resonance imaging. Nano Lett. 2006;6(10):2220-2224.

3. Jacques V, Desreux J. New Classes of MRI Contrast Agents. In: Krause W, editor. Contrast Agents I. Vol 221. Berlin/Heidelberg: Springer Verlag; 2002: 123.

4. Frullano L, Rohovec J, Peters J, Geraldes C. Structures of MRI Contrast Agents in Solution. In: Krause W, editor. Contrast Agents I. Vol 221: Berlin/Heidelberg: Springer Verlag; 2002:25.

5. Tóth É, Helm L, Merbach A. Relaxivity of MRI Contrast Agents. In: Krause W, editor. Contrast Agents I. Vol 221: Berlin/Heidelberg: Springer Verlag; 2002:61.

6. Brücher E. Kinetic Stabilities of Gadolinium(III) Chelates Used as MRI Contrast Agents. In: Krause W, editor. Contrast Agents I. Vol 221 : Berlin/Heidelberg: Springer Verlag; 2002:103.

7. Kabalka GW, Davis MA, Moss TH, et al. Gadolinium-labeled liposomes containing various amphiphilic Gd-DTPA derivatives: targeted MRI contrast enhancement agents for the liver. Magn Reson Med. 1991; 19(2):406-415.

8. Schnall M, Rosen M. Primer on imaging technologies for cancer. J Clin Oncol. 2006;24(20):3225-3233.

9. Mulder WJ, Strijkers GJ, Griffioen AW, et al. A liposomal system for contrast-enhanced magnetic resonance imaging of molecular targets. Bioconjug Chem. 2004;15(4):799-806.

10. Frullano L, Meade TJ. Multimodal MRI contrast agents. J Biol Inorg Chem. 2007;12(7):939-949.
11. Kircher MF, Mahmood U, King RS, Weissleder R, Josephson L. A multimodal nanoparticle for preoperative magnetic resonance imaging and intraoperative optical brain tumor delineation. Cancer Res. 2003;63(23):8122-8125.

12. Gianella A, Jarzyna PA, Mani V, et al. Multifunctional nanoemulsion platform for imaging guided therapy evaluated in experimental cancer. ACS Nano. 2011;5(6):4422-4433.

13. Kamaly N, Kalber T, Thanou M, Bell JD, Miller AD. Folate receptor targeted bimodal liposomes for tumor magnetic resonance imaging. Bioconjug Chem. 2009;20(4):648-655.

14. Kamaly N, Kalber T, Ahmad A, et al. Bimodal paramagnetic and fluorescent liposomes for cellular and tumor magnetic resonance imaging. Bioconjug Chem. 2008;19(1):118-129.

15. Kluza E, van der Schaft DW, Hautvast PA, et al. Synergistic targeting of alphavbeta3 integrin and galectin-1 with heteromultivalent paramagnetic liposomes for combined MR imaging and treatment of angiogenesis. Nano Lett. 2010;10(1):52-58.

16. van Tilborg GA, Mulder WJ, Chin PT, et al. Annexin A5-conjugated quantum dots with a paramagnetic lipidic coating for the multimodal detection of apoptotic cells. Bioconjug Chem. 2006;17(4): $865-868$.

17. Mulder WJ, Strijkers GJ, Habets JW, et al. MR molecular imaging and fluorescence microscopy for identification of activated tumor endothelium using a bimodal lipidic nanoparticle. FASEB J. 2005; 19(14):2008-2010.

18. Mulder WJ, van der Schaft DW, Hautvast PA, et al. Early in vivo assessment of angiostatic therapy efficacy by molecular MRI. FASEB J. 2007;21(2):378-383.

19. Mulder WJ, Koole R, Brandwijk RJ, et al. Quantum dots with a paramagnetic coating as a bimodal molecular imaging probe. Nano Lett. 2006;6(1):1-6.

20. Van Schooneveld MM, Vucic E, Koole R, et al. Improved biocompatibility and pharmacokinetics of silica nanoparticles by means of a lipid coating: a multimodality investigation. Nano Lett. 2008;8(8): 2517-2525.

21. Sun C, Lee JS, Zhang M. Magnetic nanoparticles in MR imaging and drug delivery. Adv Drug Deliv Rev. 2008;60(11):1252-1265.

22. Mulder WJ, Strijkers GJ, van Tilborg GA, Griffioen AW, Nicolay K. Lipid-based nanoparticles for contrast-enhanced MRI and molecular imaging. NMR Biomed. 2006;19(1):142-164.

23. Kamaly N, Miller AD. Paramagnetic liposome nanoparticles for cellular and tumour imaging. Int J Mol Sci. 2010;11(4):1759-1776.

24. Mulder WJ, Strijkers GJ, van Tilborg GA, Cormode DP, Fayad ZA, Nicolay K. Nanoparticulate assemblies of amphiphiles and diagnostically active materials for multimodality imaging. Acc Chem Res. 2009; 42(7):904-914.

25. Kostarelos K, Miller AD. Synthetic, self-assembly ABCD nanoparticles; a structural paradigm for viable synthetic non-viral vectors. Chem Soc Rev. 2005;34(11):970-994.

26. Xia W, Low PS. Folate-targeted therapies for cancer. $J$ Med Chem. 2010;53(19):6811-6824.

27. Sega EI, Low PS. Tumor detection using folate receptor-targeted imaging agents. Cancer Metastasis Rev. 2008;27(4):655-664.

28. Xiang G, Wu J, Lu Y, Liu Z, Lee RJ. Synthesis and evaluation of a novel ligand for folate-mediated targeting liposomes. Int J Pharm. 2008; 356(1-2):29-36.

29. Yoo HS, Park TG. Folate-receptor-targeted delivery of doxorubicin nano-aggregates stabilized by doxorubicin-PEG-folate conjugate. J Control Release. 2004;100(2):247-256.

30. Leamon CP, Reddy JA. Folate-targeted chemotherapy. Adv Drug Deliv Rev. 2004;56(8):1127-1141.

31. Ayyagari AL, Zhang X, Ghaghada KB, Annapragada A, Hu X, Bellamkonda RV. Long-circulating liposomal contrast agents for magnetic resonance imaging. Magn Reson Med. 2006;55(5): $1023-1029$ 
32. Unger E, Needleman P, Cullis P, Tilcock C. Gadolinium-DTPA liposomes as a potential MRI contrast agent. Work in progress. Invest Radiol. 1988;23(12):928-932.

33. Strijkers GJ, Mulder WJ, van Heeswijk RB, et al. Relaxivity of liposomal paramagnetic MRI contrast agents. MAGMA. 2005;18(4):186-192.
34. Maruyama K. PEG-liposome in DDS and clinical studies. Nippon Rinsho. 1998;56(3):632-637.

35. Muller C, Schubiger PA, Schibli R. In vitro and in vivo targeting of different folate receptor-positive cancer cell lines with a novel 99 mTc-radiofolate tracer. Eur J Nucl Med Mol Imaging. 2006;33(10): 1162-1170.

\section{Publish your work in this journal}

The International Journal of Nanomedicine is an international, peerreviewed journal focusing on the application of nanotechnology in diagnostics, therapeutics, and drug delivery systems throughout the biomedical field. This journal is indexed on PubMed Central, MedLine, CAS, SciSearch $\AA$, Current Contents ${ }^{\circledR} /$ Clinical Medicine,
Journal Citation Reports/Science Edition, EMBase, Scopus and the Elsevier Bibliographic databases. The manuscript management system is completely online and includes a very quick and fair peer-review system, which is all easy to use. Visit http://www.dovepress.com/ testimonials.php to read real quotes from published authors. 